\title{
Immunological and Therapeutic Strategies against Salmonid Cryptobiosis
}

\author{
Patrick T. K. Woo \\ Department of Integrative Biology, University of Guelph, Guelph, ON, Canada N1G 2W1 \\ Correspondence should be addressed to Patrick T. K. Woo, pwoo@uoguelph.ca
}

Received 1 August 2009; Accepted 18 September 2009

Academic Editor: Luis I. Terrazas

Copyright () 2010 Patrick T. K. Woo. This is an open access article distributed under the Creative Commons Attribution License, which permits unrestricted use, distribution, and reproduction in any medium, provided the original work is properly cited.

Salmonid cryptobiosis is caused by the haemoflagellate, Cryptobia salmositica. Clinical signs of the disease in salmon (Oncorhynchus spp.) include exophthalmia, general oedema, abdominal distension with ascites, anaemia, and anorexia. The disease-causing factor is a metalloprotease and the monoclonal antibody (mAb-001) against it is therapeutic. MAb-001 does not fix complement but agglutinates the parasite. Some brook charr, Salvelinus fontinalis cannot be infected (Cryptobia-resistant); this resistance is controlled by a dominant Mendelian locus and is inherited. In Cryptobia-resistant charr the pathogen is lysed via the Alternative Pathway of Complement Activation. However, some charr can be infected and they have high parasitaemias with no disease (Cryptobia-tolerant). In infected Cryptobia-tolerant charr the metalloprotease is neutralized by a natural antiprotease, $\alpha 2$ macroglobulin. Two vaccines have been developed. A single dose of the attenuated vaccine protects $100 \%$ of salmonids (juveniles and adults) for at least 24 months. Complement fixing antibody production and cell-mediated response in vaccinated fish rise significantly after challenge. Fish injected with the DNA vaccine initially have slight anaemias but they recover and have agglutinating antibodies. On challenge, DNA-vaccinated fish have lower parasitaemias, delayed peak parasitaemias and faster recoveries. Isometamidium chloride is therapeutic against the pathogen and its effectiveness is increased after conjugation to antibodies.

\section{Introduction}

Fish has been and will continue to be one of the major sources of animal protein for humans. It will likely become more important as the population heads towards 8 billion in about 20 years as food production (e.g., growing of crops, breeding of domestic animals) has and will continue to compete with other human activities (e.g., transportation, housing, industry) for the limited usable/inhabitable land. Besides being a more affordable animal protein many species of marine fishes have beneficial health components which include the polyunsaturated fatty acids (e.g., Omega 3). However, the capture-fishery is either stagnant or has been in decline as natural fish stocks in many parts of the world have been reduced significantly because of over and/or indiscriminate fishing and/or the destruction of spawning grounds. Many undesirable discharges (e.g., organophosphates, heavy metals) into the aquatic environments, especially from industries, are known to reduce fish survival and reproduction. In some areas fish are no longer suitable for human consumption because of the high levels of accumulated pollutants, and no new fishing grounds have been discovered. According to the Food and Agriculture Organization, aquaculture continues to be the fastest food producing sector with about a $10 \%$ annual increase. It would be higher if not for disease outbreaks [1]. Intensive culture of freshwater and marine fishes in cages is well developed in many countries, especially in those that have large numbers of rivers and lakes and/or long coastlines (e.g., China, Chile, Norway). However, disease outbreaks become more frequent as intensive fish culture tends to facilitate the transmission of parasites between fish in cages and the acquisition of pathogens from feral fishes that are attracted to the uneaten food in cages [2].

The piscine immune system is well developed, and in many ways it is similar to that in mammals (e.g., [3]) which include a comparable set of immunocompetent cells [4]. In general, the adaptive immune response is slower to 


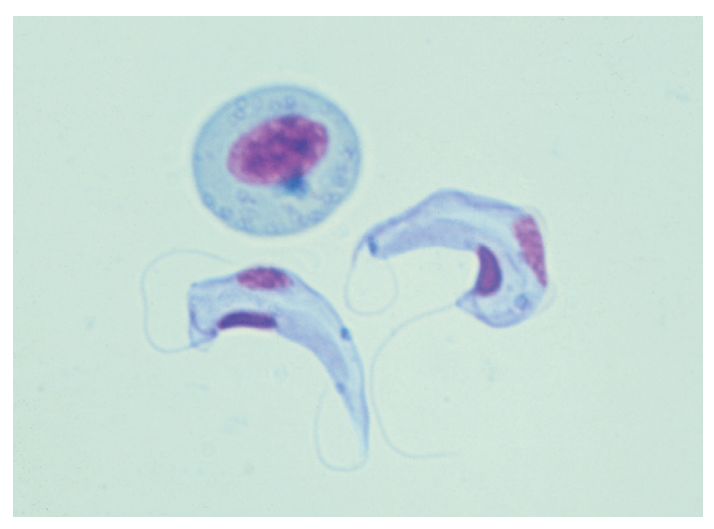

FIGURE 1: Cryptobia salmositica with red cell from an experimentally infected rainbow trout (reproduced from Woo [5]).

develop in fish than in mammals, and this is in part due to its lower body temperature. However, the innate immunity in fish is as well developed and is as responsive as that in mammals. The present discussion is in two parts; the first part (Section 2) is a brief review on Cryptobia and the pathobiology in cryptobiosis-information which are relevant to the discussion on the development of strategies against the pathogen and disease (Section 3).

\section{Cryptobia salmositica and Cryptobiosis}

2.1. The Parasite. Cryptobia is a parasitic flagellate that has worldwide distribution, and a few species are known to cause disease in marine and freshwater fishes. This extracellular parasite is elongated and is a little larger than a fish red blood cell. Its nucleus is close to the kinetoplast which is located at the anterior end. The parasite has an anterior flagellum and a recurrent flagellum that attaches to the body and exit as a free flagellum at the posterior end [5].

Salmonid cryptobiosis is caused by the haemoflagellate Cryptobia (Trypanoplasma) salmositica (Figure 1). The pathogen has been reported in all species of Pacific salmon, Oncorhynchus spp., along the west coast of North America [5], and outbreaks of cryptobiosis with high fish mortalities have occurred in both freshwater hatcheries and in sea cage cultures [6]. The parasite multiplies by binary fission, and the parasitaemia peaks at about 4-5 weeks after infection (e.g., [7-9]). The severity of the disease (e.g., the anaemia) is directly related to the parasitaemia and clinical signs include exophthalmia (Figure 2), general oedema, abdominal distension with ascites, a microcytic and hypochromic anaemia, positive antiglobulin reaction (or positive Coombs' test) of red cells (Figure 3), and anorexia $[7,10,11]$. Anorexia is a double-edged sword-it is beneficial to the host in that it reduces the severity of the disease by lowering plasma proteins and subsequently the parasitaemia but it is also detrimental to the fish in that it contributes to the immunodepression [11, 12]. During acute disease the haemolytic activity of complement is significantly lowered [13]. In addition, plasma thyroxine (T3 and T4),

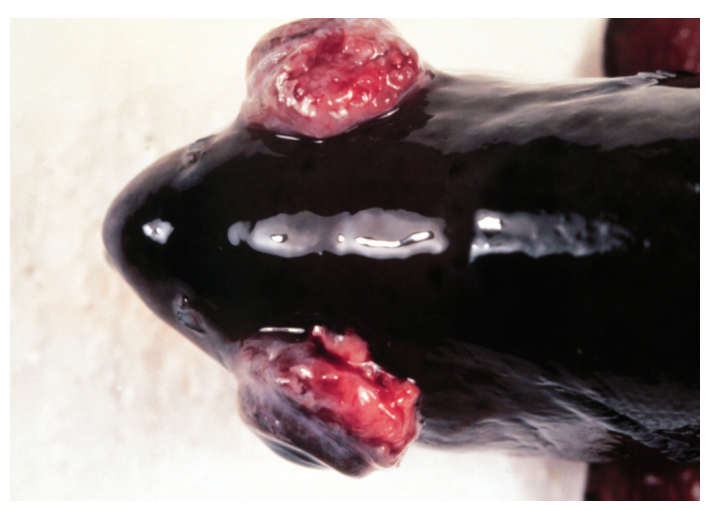

FIGURE 2: Exophthalmia in rainbow trout with an acute experimental cryptobiosis (reproduced from Woo [5]).

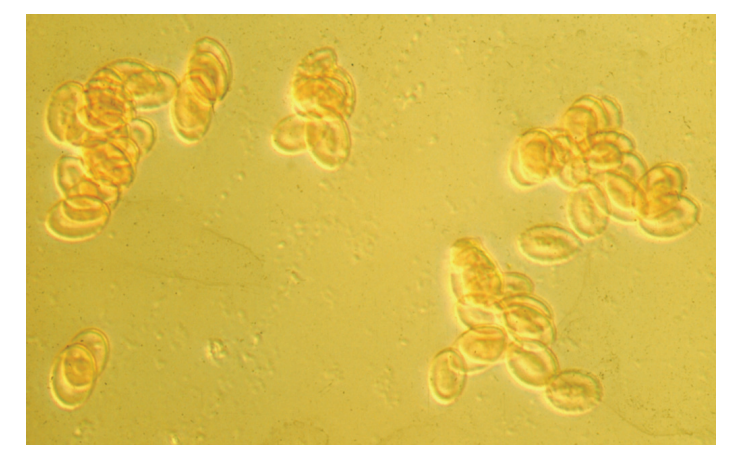

FIGURE 3: Positive antiglobulin reaction; red blood cells from an experimentally infected rainbow trout (reproduced from Woo [5]).

protein, and glucose are reduced along with depletion of liver glycogen [14]. The metabolism and swimming performance of infected rainbow trout are also significantly reduced [15], and the bioenergetic cost of the disease in juvenile fish is considerable. These are contributing factors to the retarded growth as there are significant reductions in food consumption, dry weight and energy gained, energy concentration, and gross conversion efficiency. However, the attenuated vaccine strain (Section 3.3.1) has no detectable bioenergetic cost to juvenile fish [16].

2.2. A Cysteine Protease and a Metalloprotease. Two proteases have been identified in the pathogen [17]. The cysteine protease consisting of four polypeptide bands $(49,60,66$, and $97 \mathrm{kDa}$ ) is a metabolic enzyme while the metalloprotease $(200 \mathrm{kDa})$ is a histolytic enzyme. The metalloprotease has been isolated and purified (Figure 4). Its proteolytic activity is inhibited by excess of zinc ions [17-19]. The purified enzyme lyses red blood cells [20] by digesting erythrocyte membranes [18]. Consequently, it is an important contributing factor to the anaemia which is a very consistent clinical sign of the disease. Also, the purified metalloprotease readily degrades types I, IV, and V collagens (Figure 5) and laminin [18]. It is secreted by the parasite in fish [21] and in culture [19], and it contributes significantly to the development 


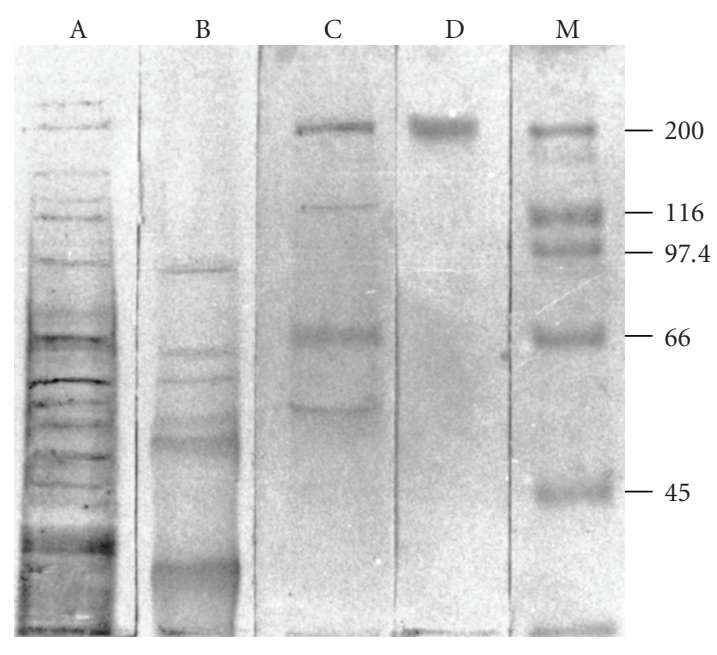

Figure 4: Purification of cysteine protease and metalloprotease from Cryptobia salmositica. Lane A: crude cell lysate; lane B: partially purified cysteine protease from a DEAE-agarose column; lane C: partially purified metalloprotease from a DEAE-agarose column; lane D: a single band of purified metalloprotease from a Sephacryl S-300 column; lane M: molecular markers (kDa) (reproduced from Zuo \& Woo [18]).

of the disease and histopathogical lesions in infected fish [22].

Briefly, metalloprotease activities can be neutralized by either a monoclonal antibody (Section 3.1) or a natural antiprotease (Section 3.2.2) or the antibody against the DNA vaccine (Section 3.3.2); this neutralization essentially "disarms" the pathogen so that the host immune system can more readily control the infection.

\section{Strategies against Cryptobia and Cryptobiosis}

3.1. Serological. A murine IgG1 monoclonal antibody (mAb001; Figure 6) has been produced against the $200 \mathrm{kDa}$ glycoprotein (Cs-gp200). The antibody is therapeutic when injected intraperitoneally into infected fish - it significantly lowers the parasitaemias in fish and this is similar to the effects of the inoculation of antisera from fish that had recovered from cryptobiosis. Also, $\mathrm{mAb}-001$ has prophylactic effects in fish [23]; however, it does not fix complement but agglutinates live parasite. In vitro exposure of the parasite to $\mathrm{mAb}-001$ reduces its survival and infectivity when inoculated back into fish [24]. The monoclonal antibody also inhibits parasite multiplication and its aerobic respiration [25], and it completely neutralizes the activity of the metalloprotease [26]. The Cs-gp200 epitope consists of carbohydrate determinants and conformational polypeptide with internal disulphide bonds. It is hydrophilic and is secreted by the parasite [27]. The epitope has its asparagine-bound N-glycosidically linked hybrid-type carbohydrate chain with the minimum length of a chitobiose core unit. It has a phosphatidylinositol residue which anchors the conformational polypeptide (with disulphide bonds) to the surface of the pathogen. The

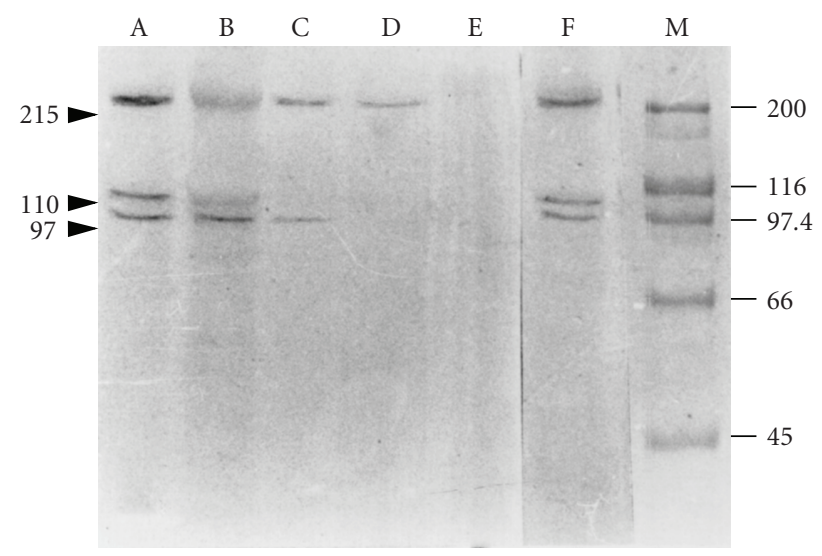

Figure 5: In vitro proteolytic degradation of collagen type $\mathrm{V}$ by purified metalloprotease from Cryptobia salmositica. Lanes A-E: collagen incubated with metalloprotease for $0,2,4,6$, and 8 hours respectively; lane $\mathrm{F}$ : collagen incubated under same conditions as lanes $\mathrm{A}-\mathrm{E}$, but without metalloprotease (control); lane M: molecular markers (reproduced from Zuo and Woo [18]).

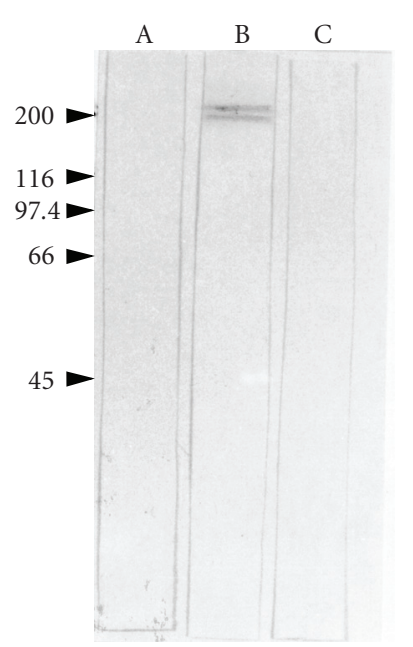

Figure 6: Immunoblot using mAb-001 on Cryptobia salmositica lysate. Lane A: mAb-001 after immunoabsorption with live parasites; lane B: mAb-001 without immunoabsorption; lane $\mathrm{C}$ : hybridoma culture medium. The numbers on the left are molecular mass $(\mathrm{kDa})$ of protein standards.

molecule is extensively posttranslationally modified [28], has high mannose components, and appears as a doublet in the pathogenic strain and as a single band in the attenuated vaccine strain [29].

3.2. Innate (Natural) Immunity. In the present discussion, a distinction is made to distinguish between the two forms of natural immunity-the first is absence of disease in an infected fish (pathogen-tolerant fish) and the second is resistance to infection by a fish (pathogen-resistant fish).

3.2.1. Cryptobia-Resistant Fish. Some laboratory/hatchery raised brook charr, Salvelinus fontinalis, cannot be infected 
with C. salmositica, and this is innate resistance to infection. Resistance to Cryptobia infection is inherited by progeny and it is controlled by a single dominant Mendelian locus. Cryptobia-susceptible brook charr are homozygous recessive while the Cryptobia-resistant fish are either homozygous or heterozygous dominant for the locus [30]. Consequently, we can now breed resistant fish by initially testing the freshly collected plasma from the brood fish for cryptobiacidal effects. Briefly, under in vitro conditions fresh plasma from Cryptobia-resistant charr lyse the parasite via the Alternative Pathway of Complement Activation [31]. There is no detectable difference in the immune responses of both Cryptobia-tolerant (Section 3.2.2) and Cryptobia-resistant charr to other antigenic stimulations including a commercial vaccine [32]. Not much is known about this type of resistance and its inheritance by progeny; consequently, further studies on innate resistance to infections in other animals would be most rewarding because it can be a good strategy against some pathogens.

3.2.2. Cryptobia-Tolerant Fish. Parasitaemias in some infected brook charr are just as high as those in Oncorhynchus spp.; however they do not suffer from cryptobiosis (Cryptobia-tolerant fish). Cryptobia-tolerant brook charr are resistance to disease because the metalloprotease secreted by $C$. salmositica is neutralized by the $\alpha 2$ macroglobulin (a natural antiprotease) in the blood. The amount of $\alpha 2$ macroglobulin is higher in brook charr than in rainbow trout prior to infection and it remains high (about 40\%) even at peak parasitaemia while that in trout drops to about $12 \%[21,33]$. Parasitaemias in both infected rainbow trout and brook charr peak at about 4-6 weeks after infection and as antibodies are produced the parasitaemias decline; however, the parasitaemia fluctuates in rainbow trout while that in infected Cryptobia-tolerant charr rapidly declines after peak parasitaemia [32]. Neutralization of the metalloprotease by $\alpha 2$ macroglobulin was demonstrated under both in vivo and in vitro conditions $[18,21,33]$. Since Cryptobia-tolerant charr do not suffer from clinical disease, the immune system readily controls the infection and the fish recover much more rapidly than trout from the infection [32].

An obviously option to control cryptobiosis in salmon is to consider producing transgenic Cryptobia-tolerant salmonids. It is expected that the transgenic salmon will maintain high levels of $\alpha 2$ macroglobulin in their blood, essentially to neutralize the metalloprotease secreted by the pathogen-the additional $\alpha 2$ macroglobulin will eliminate or at least reduce the severity of the disease. Since the disease is absent or less severe, the fish immune system can more effectively control the infection. This proposal is a novel approach to the management of an infectious disease in animals and it perhaps needs further discussions. An obvious "downside" with this approach is that it may increase the pool of reservoir animals (with infections but no disease) in the population but one very obvious advantage is that no further human interventions (e.g., vaccination, chemotherapy) are required once the transgenic animal is produced.

3.3. Adaptive (Acquired) Immunity. Adaptive immunity has also been exploited to protect the susceptible Oncorhynchus spp. from cryptobiosis. Two distinctly different experimental vaccines (a live attenuated vaccine and a metalloproteaseDNA vaccine) have been developed. Fish inoculated with the live attenuated Cryptobia vaccine are protected from infection when challenged with the parasite. However, the metalloprotease-DNA vaccine does not prevent an infection in vaccinated fish but antibodies produced in the vaccinated fish neutralize the disease-causing factor secreted by the pathogen. Although the DNA-vaccinated fish is infected, it does not suffer from cryptobiosis, and it essentially turns the pathogenic Cryptobia into a nonpathogenic flagellate as in the case of the Cryptobia-tolerant brook charr (Section 3.2.2).

3.3.1. Live Vaccine. C. salmositica was attenuated by prolonged in vitro culture and the strain has been cloned. The attenuated parasite is maintained in tissue culture medium and it has remained avirulent since 1990. It produces a low infection in rainbow trout, does not cause disease, circulates in the blood for at least 6 months, and is protective when the fish is challenged with the pathogen [34]. The vaccine strain is smaller in size and has lost a few polypeptides. It is adapted to in vitro culture and hence multiplies much more readily than the pathogenic strain in tissue culture medium $[35,36]$. A single injection of the strain protects $100 \%$ of vaccinated fish and consequently it is used routinely as an experimental vaccine to study the development and mechanism of protective immunity in salmonids and the pathobiology of the disease. The vaccine has no detectable bioenergetic cost to juvenile rainbow trout [16], and it protects various species of juvenile and adult salmonids from the pathogen (e.g., $[8,9,37-41])$.

Rainbow trout vaccinated in fresh water and transferred to sea water are also protected on parasite challenge [42]. A single dose of the vaccine protects rainbow trout for at least 24 months [8]. Vaccinated fish are partially protected if they are challenged at 2 weeks postvaccination (wpv) while all vaccinated fish are protected (e.g., no drop in packed cell volume and virtually no detectable infection after parasite challenge) at $4 \mathrm{wpv}$. Protection is via the production of complement fixing antibodies and under in vitro conditions activated macrophages from head kidneys of vaccinated fish show antibody-independent and antibodydependent cytotoxicities. Also, in the presence of antiserum macrophages are very efficient in engulfing living parasites (Figure 7).

The complement fixing antibody titres (e.g., [8]) and cell-mediated response (e.g., [40]) in vaccinated fish rise significantly soon after parasite challenge (classical secondary responses), and the responses in vaccinated and challenged fish are similar to those in naïve fish at 6 weeks after infection. Humoral and cell-mediated immunity are important 


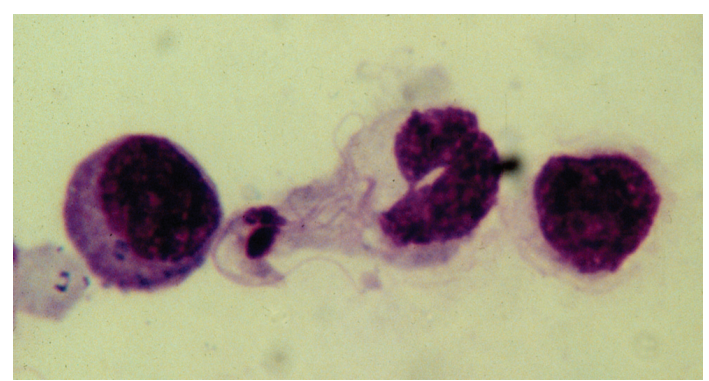

FIgURE 7: Peritoneal macrophage in the ascites of an experimentally infected rainbow trout, Cryptobia salmositica in the process of being ingested (reproduced from Woo [7]).

components of the protection against $C$. salmositica in both vaccinated and recovered fish (e.g., $[8,9,40,43]$ ).

3.3.2. Metalloprotease-DNA Vaccine. As indicated earlier (Sections 3.1 and 3.2.2) the disease causing metalloprotease ( $200 \mathrm{kDa}$ glycoprotein) can be neutralized. This is accomplished either by the $\alpha 2$ macroglobulin (a natural antiprotease) in Cryptobia-tolerant brook charr [18, 21,33] where the parasitaemia does not fluctuate and the fish recovers rapidly [32] or by an antibody (mAb-001) produced against the $200 \mathrm{kDa}$ glycoprotein [26]. The monoclonal antibody (mAb-001) agglutinates the parasite and reduces its survival and infectivity [24]. Neutralization of the metalloprotease by antibodies in vaccinated fish is the basis of our current DNA vaccine.

Briefly, the metalloprotease and cysteine protease genes of C. salmositica were sequenced $[45,46]$ and inserted into plasmid vectors (pEGFP-N) to produce a metalloproteaseplasmid vaccine and a cysteine-plasmid vaccine [47]. Rainbow trout and Atlantic salmon, Salmo salar, injected intramuscularly with the metalloprotease-plasmid vaccine consistently had lower packed cell volume (as metalloprotease was secreted into the blood) than controls (fish inoculated either with plasmid alone or with cysteine-plasmid vaccine) at $2-$ 4 wpv. However, the packed cell volume in metalloproteasevaccinated fish returned to normal by $5 \mathrm{wpv}$ - this was because the metalloprotease was neutralized as antibodies were produced. Agglutinating antibodies against $C$. salmositica were detected 5-7 wpv in the blood (and not before $5 \mathrm{wpv}$ ) in metalloprotease-vaccinated fish, but not in fish injected with either the cysteine-plasmid or plasmid alone injected fish. Fish were challenged with the pathogen at $7 \mathrm{wpv}$ and the metalloprotease-vaccinated fish had lower parasitaemia, delayed peak parasitaemia, and faster recovery than control infected fish. In a recent review on the use of DNA vaccines in aquatic organisms Kurath [48] confirms that this is the "... first published demonstration of protective effects of a fish parasite DNA vaccine in fish."

Many protozoa that are of medical and economic importance (e.g., Trypanosoma spp., Leishmania spp.) have metalloprotease and cysteine protease [49]. Some of these pathogens also modify their surface coats to evade the host immune response (e.g., antigenic variations as in the
Glossina-transmitted mammalian trypanosomes in Africa); consequently vaccines based on surface membrane epitopes are not effective. However, an enzyme-based vaccine may be worth serious considerations as enzymes are quite conserve. Also, an enzyme-based vaccine will most likely protect against all isolates of the pathogen including those from different geographical locations which may have different surface membrane antigens. Enzymes are generally quite conserve and this is true even among different pathogens; for example, sequences of PCR-derived fragments of the metalloprotease gene of C. salmositica [45] are similar to those in other kinetoplastids, such as Leishmania chagasi, L. donovani [50], Trypanosoma cruzi [51], and T. brucei [52]. Major surface protease (MSP) also known as GP63 or leishmanolysin is a highly abundant zinc metalloprotease present on the cell surface of Leishmania spp. The NCBIconserved domain search shows that the alignment for the metalloprotease of C. salmositica has $78.3 \%$ similarity with peptidase_M8, leishmanolysin domain [45].

3.4. Chemotherapy and Immunochemotherapy. Chemotherapy is essentially differential toxicity; that is, the drug is more toxic to the target organism than it is to the host. Severity of the side effects of chemotherapy is dependent partly on tissue damage and adverse reactions by the host to the drug. However, the drug can be directed more specifically to the pathogen if it is conjugated to an antibody specific for the target organism. Immunochemotherapy will obviously increase costs and is generally not meant for routine use; it may however be a useful tool under certain circumstances as it reduces the drug dosage and its side effects. For example, it can be used to treat infected brood fish as about $50 \%$ of brood fish annually die from cryptobiosis in some hatcheries on the west coast of North America [6]. It is expected the side effects and accumulation of drug residues in host tissues will be reduced in immunochemotherapy, and this may also lower the risk of the development of drug-resistance by the pathogen. Reduction in drug residue in host tissues is also an important consideration if the fish are for human consumption.

3.4.1. Chemotherapy. In tropical Africa isometamidium chloride (Samorin) is widely used against trypanosomiasis in domestic animals [53], and it is also used as a prophylactic drug against bovine trypanosomiasis [54]. In fish, Samorin $(1.0 \mathrm{mg} / \mathrm{kg}$ weight $)$ reaches peak level in the blood 2-3 weeks after intramuscular injection [55]. The drug is therapeutic against $C$. salmositica in rainbow trout during pre- and postclinical phases of the disease. However, it is not effective during acute disease partly as we believe that the drug "modifies" surface epitopes of the parasite so that they are not lysed by complement fixing antibodies [56]. The drug is more effective in infected Atlantic salmon, and at a higher dose $(2.5 \mathrm{mg} / \mathrm{kg})$ the infection is eliminated in about $30 \%$ of adult fish and significantly reduces the parasitaemias in remaining fish. Also all infected juvenile chinook salmon, Oncorhynchus tshawytscha, treated with isometamidium chloride $(1.0 \mathrm{mg} / \mathrm{kg})$ survived the disease 
TABLE 1: Infectivity of Cryptobia salmositica to chinook salmon after in vitro exposure to isometamidium chloride to polyclonal antibodies from a recovered fish (reproduced from Ardelli and Woo [59]).

\begin{tabular}{|c|c|c|c|c|c|}
\hline $\begin{array}{l}\text { Weeks } \\
\text { After } \\
\text { Infection }\end{array}$ & $\begin{array}{l}\text { Group } 1 \\
\text { PAlC }\end{array}$ & $\begin{array}{l}\text { Group } 2 \\
\text { Drug }\end{array}$ & $\begin{array}{c}\text { Group } 3 \\
\text { PAl }\end{array}$ & $\begin{array}{l}\text { Group } 4 \\
\text { Antibody }\end{array}$ & $\begin{array}{c}\text { Group } 5 \\
\text { Untreated Controls }\end{array}$ \\
\hline \multirow[t]{2}{*}{1} & $1 / 10 \bullet$ & $7 / 10$ & $0 / 10$ & $0 / 10$ & $8 / 10$ \\
\hline & $0.30 \pm 0.95 \square$ & $1.80 \pm 2.78$ & 0 & 0 & $6.60 \pm 4.72$ \\
\hline \multirow[t]{2}{*}{2} & $1 / 10$ & $6 / 10$ & $2 / 10$ & $0 / 10$ & $10 / 10$ \\
\hline & $0.30 \pm 0.95$ & $8.10 \pm 5.28$ & $1.80 \pm 3.91$ & 0 & $48,750 \pm 45,814$ \\
\hline \multirow[t]{2}{*}{3} & $2 / 10$ & $7 / 10$ & $2 / 10$ & $0 / 10$ & $10 / 10$ \\
\hline & $0.50 \pm 1.27$ & $33,250 \pm 38,207$ & $1,950 \pm 4,310$ & 0 & $35,625 \pm 29,978$ \\
\hline \multirow[t]{2}{*}{4} & $2 / 10$ & $8 / 10$ & $3 / 10$ & $3 / 10$ & $10 / 10$ \\
\hline & $0.30 \pm 0.675$ & $302,000 \pm 254,142$ & $2,500 \pm 5,270$ & $5,558 \pm 16,665$ & $5,487,500 \pm 5,439,838$ \\
\hline \multirow[t]{2}{*}{5} & $2 / 10$ & $10 / 10$ & $4 / 10$ & $4 / 10$ & $10 / 10$ \\
\hline & $0.60 \pm 1.58$ & $9,395,000 \pm 16,925,911$ & $54,740 \pm 112,499$ & $556,944 \pm 1,500,001$ & $3,175,000 \pm 3,639,196$ \\
\hline \multirow[t]{2}{*}{6} & $1 / 10$ & $10 / 10$ & $4 / 10$ & $4 / 10$ & $10 / 10$ \\
\hline & $3,750 \pm 11,858$ & $13,475,000 \pm 15,298,624$ & $503,750 \pm 1,030,810$ & $2,600,000 \pm 5,577,465$ & $3,243,750 \pm 5,416,196$ \\
\hline \multirow[t]{2}{*}{7} & $1 / 10$ & $10 / 10$ & $4 / 10$ & $4 / 10$ & $10 / 10$ \\
\hline & $11,250 \pm 35,575$ & $18,305,000 \pm 52,500,000$ & $928,860 \pm 1,326,575$ & $44,383,334 \pm 129,150,260$ & $27,051,250 \pm 56,209,714$ \\
\hline
\end{tabular}

- Number of infected fish/number of fish inoculated.

Mean parasitaemia \pm standard deviation, determined by HCT or haemacytometer.

while $100 \%$ of untreated infected fish died with massive parasitaemias. The drug also has prophylactic value, and it does not seem to affect fish growth, food consumption, blood complement levels, or haematocrit values in fish [57].

Samorin accumulates rapidly in the kinetoplast of the parasite [44], causes condensation of its kinetoplast DNA, forms vacuoles, and swells the mitochondrial cristae (Figure 8). Although the parasite normally undergoes aerobic respiration [25], it also has glycolytic enzymes sequestered in microbodies called glycosomes [58]. The in vitro oxygen consumption and carbon dioxide production decrease significantly after drug exposure with very significant increases in secretion of glycolytic products (lactate and pyruvate) as the parasite switches from aerobic respiration to glycolysis after its mitochondrion is damaged by the drug [44]. Also, in vitro exposure to sublethal levels of the drug reduces infectivity of the parasite to fish and changes the surface glycoprotein antibody-receptor sites of the parasite. This alteration of surface epitopes explains the protection of some parasites from lysis by complement fixing antibodies when rainbow trout with acute infections were treated with the drug [56].

3.4.2. Immunochemotherapy. Ardelli and Woo [59] conjugated isometamidium chloride to polyclonal antibodies (from "recovered" fish) and the monoclonal antibody (mAb001, Section 3.1). The conjugated drug is on the entire parasite while the unconjugated drug accumulates only in the kinetoplast (Figure 9). Before drug conjugation both antibodies agglutinate living parasites but they react differently after drug conjugation. Polyclonal antibodies-conjugated drug (PAIC) lyses most of the parasite and it no longer agglutinates the parasite. In contrast, the $\mathrm{mAb}-00$-conjugated drug does not lyse C. salmositica but agglutinates it. After in vitro

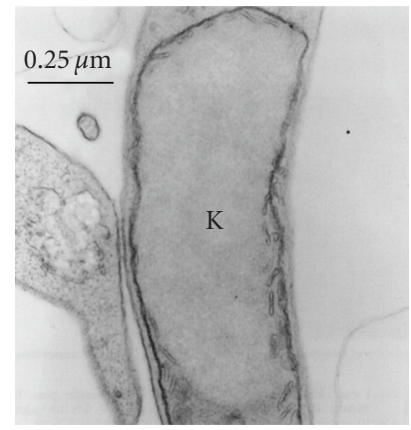

(a)

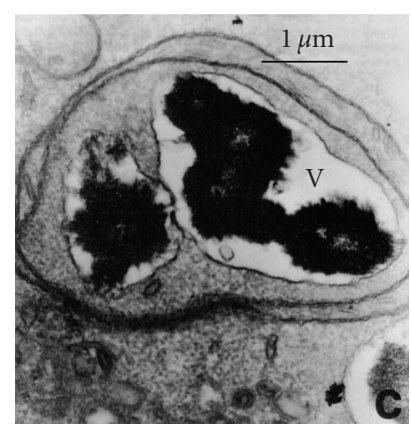

(c)

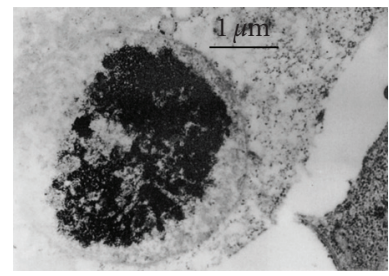

(b)

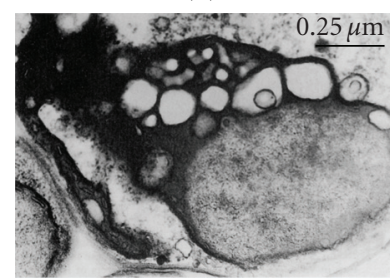

(d)

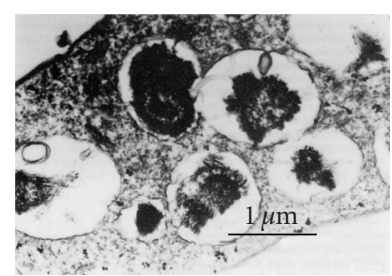

(e)
Figure 8: Ultrastructural lesions in Cryptobia salmositica after in vitro exposure to isometamidium chloride. (a) Parasite kinetoplast (K) not exposed to the drug; (b) condensation of kinetoplast DNA after exposure to the drug; (c) vacuole (V) formation after drug exposure; (d) swelling of mitochondrial cristae (C) after drug exposure; (e) vacuole formation in cytoplasm after drug exposure (reproduced from Ardelli and Woo [44]).

exposure to PAIC the infectivity of the parasite and subsequent parasitaemias in inoculated fish were significantly 


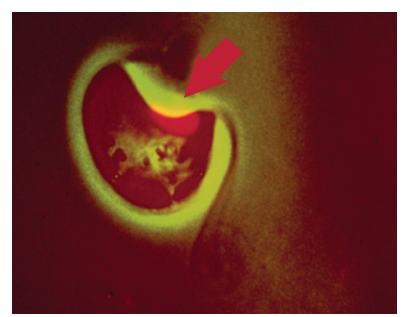

(a)

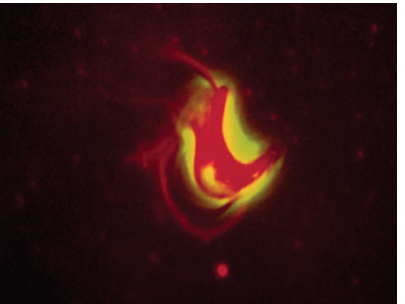

(b)

Figure 9: Phase contrast and fluorescent microscopy of Cryptobia salmositica after exposure to isometamidium chloride. (a): Exposure to drug only, note accumulation of drug (in red) in the kinetoplast; (b): exposure to drug conjugated to polyclonal antibodies from a recovered fish, note that the drug (in red) is throughout the organism (reproduced from Ardelli and Woo [59]).

lowered. Fish survival (Table 1) was much higher in juvenile chinook salmon infected with parasites exposed to the polyclonal antibodies-conjugated drug (PAIC) than to drug alone (Drug) or to polyclonal antibodies alone (Antibody) or to drug plus polyclonal antibody (PAI). Also, preliminary studies indicate the drug-antibody conjugate to be effective when injected into infected fish. The results are encouraging and further studies are needed, for example, to determine dosages needed, refinement of the approach (e.g., stage of infection, species of salmonids).

\section{Conclusions}

Our concerted efforts to better understand the biology of Cryptobia and the mechanism of the disease have allowed us to develop more rational strategies against the pathogen and disease. We have been relatively successful in exploiting the piscine immune system to protect salmonids against $C$. salmositica and cryptobiosis. This is an ongoing and evolving research program and there is obviously a great deal of work that needs to be conducted. However, I hope our research will be of interest and perhaps be useful to colleagues who are also developing control measures against similar pathogenic organisms. The research has been both challenging and fascinating, and I would like to think the best is yet to come.

\section{Acknowledgments}

The author acknowledges the valuable contributions of a good number of talented and highly dedicated graduate students and colleagues who had over the years joined him in this delightful and exciting venture. Obviously, the program would not have been possible without financial support. Consequently, the author is most grateful to the Natural Science and Engineering Research Council (NSERC) and the Department of Fisheries and Oceans, Canada, for their continued support over the years.

\section{References}

[1] P. T. K. Woo, "Protective immunity in fish against protozoan diseases," Parassitologia, vol. 49, no. 3, pp. 185-191, 2007.
[2] P. T. K. Woo, D. W. Bruno, and L. H. S. Lim, Diseases and Disorders of Finfish in Cage Culture, CABI, Wallingford, UK, 2002.

[3] W. B. Van Muiswinkel and B. Vervoorn-Van Der Wal, "The immune system of fish," in Fish Diseases and Disorders, Volume 1: Protozoan and Metazoan Infections, P. T. K. Woo, Ed., pp. 678-701, CABI, Wallingford, UK, 2nd edition, 2006.

[4] B. F. Ardelli and P. T. K. Woo, "Immunocompetent cells and their mediators in Fin Fish," in Fish Diseases and Disorders, Volume 1: Protozoan and Metazoan Infections, P. T. K. Woo, Ed., pp. 702-724, CABI, Wallingford, UK, 2nd edition, 2006.

[5] P. T. K. Woo, "Cryptobia (Trypanoplasma) salmositica and salmonid cryptobiosis," Journal of Fish Diseases, vol. 26, no. 11-12, pp. 627-646, 2003.

[6] P. T. K. Woo, "Diplomonadida (Phylum Parabasalia) and Kinetoplastea (Phylum Euglenozoa)," in Fish Diseases and Disorders, Volume 1: Protozoan and Metazoan Infections, P. T. K. Woo, Ed., pp. 46-115, CABI, Wallingford, UK, 2nd edition, 2006.

[7] P. T. K. Woo, "Trypanoplasma salmositica: experimental infections in rainbow trout, Salmo gairdneri," Experimental Parasitology, vol. 47, no. 1, pp. 36-48, 1979.

[8] S. Li and P. T. K. Woo, "Efficacy of a live Cryptobia salmositica vaccine, and the mechanism of protection in vaccinated rainbow trout, Oncorhynchus mykiss, against cryptobiosis," Veterinary Immunology and Immunopathology, vol. 48, no. 3-4, pp. 343-353, 1995.

[9] B. F. Ardelli and P. T. K. Woo, "Experimental Cryptobia salmositica (Kinetoplastida) infections in Atlantic salmon, Salmo salar L.: cell-mediated and humoral immune responses against the pathogenic and vaccine strains of the parasite," Journal of Fish Diseases, vol. 25, no. 5, pp. 265-274, 2002.

[10] S. Li and P. T. K. Woo, "Anorexia reduces the severity of cryptobiosis in Oncorhynchus mykiss," Journal of Parasitology, vol. 77, no. 3, pp. 467-471, 1991.

[11] P. T. Thomas and P. T. K. Woo, "Anorexia in Oncorhynchus mykiss (Walbaum) infected with Cryptobia salmositica (Sarcomastigophora, Kinetoplastida), its onset and contribution to the immunodepression," Journal of Fish Diseases, vol. 15, pp. 443-447, 1992.

[12] S. R. M. Jones, P. T. K. Woo, and R. M. W. Stevenson, "Immunosuppression in rainbow trout, Salmo gairdneri Richardson, caused by the haemoflagellate Cryptobia salmositica Katz, 1951," Journal of Fish Diseases, vol. 9, no. 5, pp. 431438, 1986.

[13] P. T. Thomas and P. T. K. Woo, "Complement activity in Salmo gairdneri Richardson infected with Cryptobia salmositica and its relationship to the anaemia in cryptobiosis," Journal of Fish Diseases, vol. 12, pp. 395-397, 1989.

[14] C. W. Laidley, P. T. K. Woo, and J. F. Leatherland, "The stress response of rainbow trout to experimental infection with the blood parasite, Cryptobia salmositica Katz, 1951," Journal of Fish Biology, vol. 32, pp. 253-261, 1988.

[15] A. K. Kumaraguru, F. W. Beamish, and P. T. K. Woo, "Impact of a pathogenic haemoflagellate, Cryptobia salmositica Katz, on the metabolism and swimming performance of rainbow trout, Oncorhynchus mykiss (Walbaum)," Journal of Fish Diseases, vol. 18, no. 4, pp. 297-305, 1995.

[16] F. W. H. Beamish, A. Sitja-Bobadilla, J. A. Jebbink, and P. T. $\mathrm{K}$. Woo, "Bioenergetic cost of cryptobiosis in fish: rainbow trout Oncorhynchus mykiss infected with Cryptobia salmositica and with an attenuated live vaccine," Diseases of Aquatic Organisms, vol. 25, no. 1-2, pp. 1-8, 1996. 
[17] X. Zuo and P. T. K. Woo, "Proteases in pathogenic and nonpathogenic haemoflagellates, Cryptobia spp. (Sarcomastigophora: Kinetoplastida), of fishes," Disease of Aquatic Organisms, vol. 29, no. 1, pp. 57-65, 1997.

[18] X. Zuo and P. T. K. Woo, "Purified metalloprotease from the pathogenic haemoflagellate, Cryptobia salmositica, and its in vitro proteolytic activities," Diseases of Aquatic Organisms, vol. 30, pp. 177-185, 1997.

[19] X. Zuo and P. T. K. Woo, "Characterization of purified metallo- and cysteine proteases from the pathogenic haemoflagellate Cryptobia salmositica Katz 1951," Parasitology Research, vol. 84, no. 6, pp. 492-498, 1998.

[20] X. Zuo and P. T. K. Woo, "In vitro haemolysis of piscine erythrocytes by purified metallo-protease from the pathogenic haemoflagellate, Cryptobia salmositica Katz," Journal of Fish Diseases, vol. 23, no. 3, pp. 227-230, 2000.

[21] X. Zuo and P. T. K. Woo, "In vivo neutralization of Cryptobia salmositica metallo-protease by $\alpha 2$-macroglobulin in the blood of rainbow trout Oncorhynchus mykiss and in brook charr Salvelinus fontinalis," Disease of Aquatic Organisms, vol. 29, no. 1, pp. 67-72, 1997.

[22] M. Bahmanrokh and P. T. K. Woo, "Relations between histopathology and parasitaemias in Oncorhynchus mykiss infected with Cryptobia salmositica, a pathogenic haemoflagellate," Diseases of Aquatic Organisms, vol. 46, no. 1, pp. 41-45, 2001.

[23] S. Feng and P. T. K. Woo, "Therapeutic and prophylactic effects of a protective monoclonal antibody (MAb-001) against the pathogenic haemoflagellate Cryptobia salmositic," Disease of Aquatic Organisms, vol. 28, no. 3, pp. 211-219, 1997.

[24] S. Feng and P. T. K. Woo, "Biological characterization of a monoclonal antibody against a surface membrane antigen on Cryptobia salmositica Katz, 1951," Journal of Fish Diseases, vol. 19, no. 2, pp. 137-143, 1996.

[25] N. Hontzeas, S. Feng, and P. T. K. Woo, "Inhibitory effects of a monoclonal antibody (MAb-001) on in vitro oxygen consumption and multiplication of the pathogenic haemoflagellate, Cryptobia salmositica Katz," Journal of Fish Diseases, vol. 24, no. 7, pp. 391-398, 2001.

[26] X. Zuo, S. Feng, and P. T. K. Woo, "The in vitro inhibition of proteases from Cryptobia salmositica Katz by a monoclonal antibody (MAb-001) against a glycoprotein on the pathogenic haemoflagellate," Journal of Fish Diseases, vol. 20, no. 6, pp. 419-426, 1997.

[27] S. Feng and P. T. K. Woo, "Characterization of a 200 kDa glycoprotein (Cs-gp200) on the pathogenic piscine haemoflagellate Cryptobia salmositica," Diseases of Aquatic Organisms, vol. 32, no. 1, pp. 41-48, 1998.

[28] S. Feng and P. T. K. Woo, "Biochemical characterisation of an epitope on the surface membrane antigen (Cs-gp200) of the pathogenic piscine haemoflagellate Cryptobia salmositica Katz 1951," Experimental Parasitology, vol. 88, no. 1, pp. 3-10, 1998.

[29] S. Feng and P. T. K. Woo, "Cell membrane glycoconjugates on virulent and avirulent strains of the haemoflagellate Cryptobia salmositica (Kinetoplastida)," Journal of Fish Diseases, vol. 24, no. 1, pp. 23-32, 2001.

[30] G. M. Forward, M. M. Ferguson, and P. K. T. Woo, "Susceptibility of brook charr, Salvelinus fontinalis to the pathogenic haemoflagellate, Cryptobia salmositica, and the inheritance of innate resistance by progenies of resistant fish," Parasitology, vol. 111, no. 3, pp. 337-345, 1995.

[31] G. M. Forward and P. T. K. Woo, "An in vitro study on the mechanism of innate immunity in Cryptobia-resistant brook char (Salvelinus fontinalis) against Cryptobia salmositica," Parasitology Research, vol. 82, no. 3, pp. 238-241, 1996.

[32] B. F. Ardelli and P. T. K. Woo, "Immune response of Cryptobiaresistant and Cryptobia-susceptible Salvelinus fontinalis to an Aeromonas salmonicida vaccine," Diseases of Aquatic Organisms, vol. 23, no. 1, pp. 33-38, 1995.

[33] X. Zuo and P. T. K. Woo, "Natural anti-proteases in rainbow trout, Oncorhynchus mykiss and brook charr, Salvelinus fontinalis and the in vitro neutralization of fish $\alpha 2$-macroglobulin by the metalloprotease from the pathogenic haemoffagellate, Cryptobia salmositica," Parasitology, vol. 114, no. 4, pp. 375382, 1997.

[34] P. T. K. Woo and S. Li, "In vitro attenuation of Cryptobia salmositica and its use as a live vaccine against cryptobiosis in Oncorhynchus mykiss," Journal of Parasitology, vol. 76, no. 5, pp. 752-755, 1990.

[35] P. T. K. Woo and P. T. Thomas, "Polypeptide and antigen profiles of Cryptobia salmositica, C. bullocki and C. catostomi (Kinetoplastida, Sarcomastigophora) isolated from fishes," Diseases of Aquatic Organisms, vol. 11, pp. 201-205, 1991.

[36] P. T. K. Woo and P. T. Thomas, "Comparative in vitro studies on virulent and avirulent strains of Cryptobia salmositica Katz, 1951 (Sarcomastigophora, Kinetoplastida)," Journal of Fish Diseases, vol. 15, pp. 261-266, 1992.

[37] A. Sitja-Bobadilla and P. T. K. Woo, "An enzyme-linked immunosorbent assay (ELISA) for the detection of antibodies against the pathogenic haemoflagellate, Cryptobia salmositica Katz, and protection against cryptobiosis in juvenile rainbow trout, Oncorhynchus mykiss (Walbaum), inoculated with a live vaccine," Journal of Fish Diseases, vol. 17, no. 4, pp. 399-408, 1994.

[38] S. Feng and P. T. K. Woo, "Complement fixing antibody production in thymectomized Oncorhynchus mykiss, vaccinated against or infected with the pathogenic haemoflagellate Cryptobia salmositica," Folia Parasitologica, vol. 44, no. 3, pp. 188-194, 1997.

[39] S. Feng and P. T. K. Woo, "In vitro and in vivo effects of rabbit anti-thymocyte serum on circulating leucocytes and production of complement fixing antibodies in thymectomized Oncorhynchus mykiss (Walbaum) infected with Cryptobia salmositica Katz, 1951," Journal of Fish Diseases, vol. 21, no. 4, pp. 241-248, 1998.

[40] M. Mehta and P. T. K. Woo, "Acquired cell-mediated protection in rainbow trout, Oncorhynchus mykiss, against the haemoflagellate, Cryptobia salmositica," Parasitology Research, vol. 88, no. 11, pp. 956-962, 2002.

[41] A. Chin, B. D. Glebe, and P. T. K. Woo, "Humoral response and susceptibility of five full-sib families of Atlantic salmon, Salmo salar L., to the haemoflagellate, Cryptobia salmositica," Journal of Fish Diseases, vol. 27, no. 8, pp. 471-481, 2004.

[42] S. Li and P. T. K. Woo, "Vaccination of rainbow trout, Oncorhynchus mykiss (Walbaum), against cryptobiosis: efficacy of the vaccine in fresh and sea water," Journal of Fish Diseases, vol. 20, no. 5, pp. 369-374, 1997.

[43] S. Feng and P. T. K. Woo, "Cell-mediated immune response and T-like cells in thymectomized Oncorhynchus mykiss (Walbaum) infected with or vaccinated against the pathogenic haemoflagellate Cryptobia salmositica Katz, 1951," Parasitology Research, vol. 82, no. 7, pp. 604-611, 1996.

[44] B. F. Ardelli and P. T. K. Woo, "The in vitro effects of isometamidium chloride (Samorin) on the piscine hemoflagellate Cryptobia salmositica (Kinetoplastida, Bodonina)," Journal of Parasitology, vol. 87, no. 1, pp. 194-202, 2001. 
[45] P. R. R. Jesudhasan, C.-W. Tan, and P. T. K. Woo, "A metalloproteinase gene from the pathogenic piscine hemoflagellate, Cryptobia salmositica," Parasitology Research, vol. 100, no. 4, pp. 899-904, 2007.

[46] P. R. R. Jesudhasan, C. W. Tan, N. Hontzeas, and P. T. K. Woo, "A cathepsin L-like cysteine proteinase gene from the pathogenic piscine hemoflagellate, Cryptobia salmositica," Parasitology Research, vol. 100, pp. 881-886, 2007.

[47] C.-W. Tan, P. R. R. Jesudhasan, and P. T. K. Woo, “Towards a metalloprotease-DNA vaccine against piscine cryptobiosis caused by Cryptobia salmositica," Parasitology Research, vol. 102, no. 2, pp. 265-275, 2008.

[48] G. Kurath, "Biotechnology and DNA vaccines for aquatic organisms," Revue Scientifique et Technique de l'Office International des Epizooties, vol. 27, pp. 175-196, 2008.

[49] P. J. Rosenthal, "Proteases of protozoan parasites," Advances in Parasitology, vol. 43, pp. 105-159, 1999.

[50] S. C. Roberts, K. G. Swihart, M. W. Agey, R. Ramamoorthy, M. E. Wilson, and J. E. Donelson, "Sequence diversity and organization of the msp gene family encoding gp63 of Leishmania chagasi," Molecular and Biochemical Parasitology, vol. 62, no. 2, pp. 157-172, 1993.

[51] N. M. El-Sayed, P. J. Myler, D. C. Bartholomeu, et al., "The genome sequence of Trypanosoma cruzi, etiologic agent of chagas disease," Science, vol. 309, no. 5733, pp. 409-415, 2005.

[52] D. J. LaCount, A. E. Gruszynski, P. M. Grandgenett, J. D. Bangs, and J. E. Donelson, "Expression and function of the Trypanosoma brucei major surface protease (GP63) genes," Journal of Biological Chemistry, vol. 278, no. 27, pp. 2465824664, 2003.

[53] L. D. B. Kinabo, J. A. Bogan, Q. A. McKellar, and M. Murray, "Relay bioavailability and toxicity of isometamidium residues: a model for human risk assessment," Veterinary and Human Toxicology, vol. 31, no. 5, pp. 417-421, 1989.

[54] L. D. Kinabo and J. A. Bogan, "Binding of isometamidium to calf thymus DNA and lipids: pharmacological implications," Journal of Veterinary Pharmacology and Therapeutics, vol. 10, no. 4, pp. 357-362, 1987.

[55] B. F. Ardelli and P. T. K. Woo, "An antigen-capture enzymelinked immunosorbent assay (ELISA) to detect isometamidium chloride in Oncorhynchus spp," Diseases of Aquatic Organisms, vol. 39, no. 3, pp. 231-236, 2000.

[56] B. F. Ardelli and P. T. K. Woo, "The therapeutic use of isometamidium chloride against Cryptobia salmositica in rainbow trout Oncorhynchus mykiss," Diseases of Aquatic Organisms, vol. 37, no. 3, pp. 195-203, 1999.

[57] B. F. Ardelli and P. T. K. Woo, "Therapeutic and prophylactic effects of isometamidium chloride (Samorin) against the hemoflagellate Cryptobia salmositica in chinook salmon (Oncorhynchus tshawytscha) and the effects of the drug on uninfected rainbow trout (O. mykiss)," Parasitology Research, vol. 87 , no. 1, pp. 18-26, 2001.

[58] B. F. Ardelli, J. D. S. Witt, and P. T. K. Woo, "The identification of glycosomes and metabolic end products in pathogenic and nonpathogenic strains of Cryptobia salmositica (Kinetoplastida: Bodonidae)," Diseases of Aquatic Organisms, vol. 42, no. 1, pp. 41-51, 2000.

[59] B. F. Ardelli and P. T. K. Woo, "Conjugation of isometamidium chloride to antibodies and the use of the conjugate against the haemoflagellate, Cryptobia salmositica Katz, 1951: an immuno-chemotherapeutic strategy," Journal of Fish Diseases, vol. 24 , no. 8, pp. 439-451, 2001. 

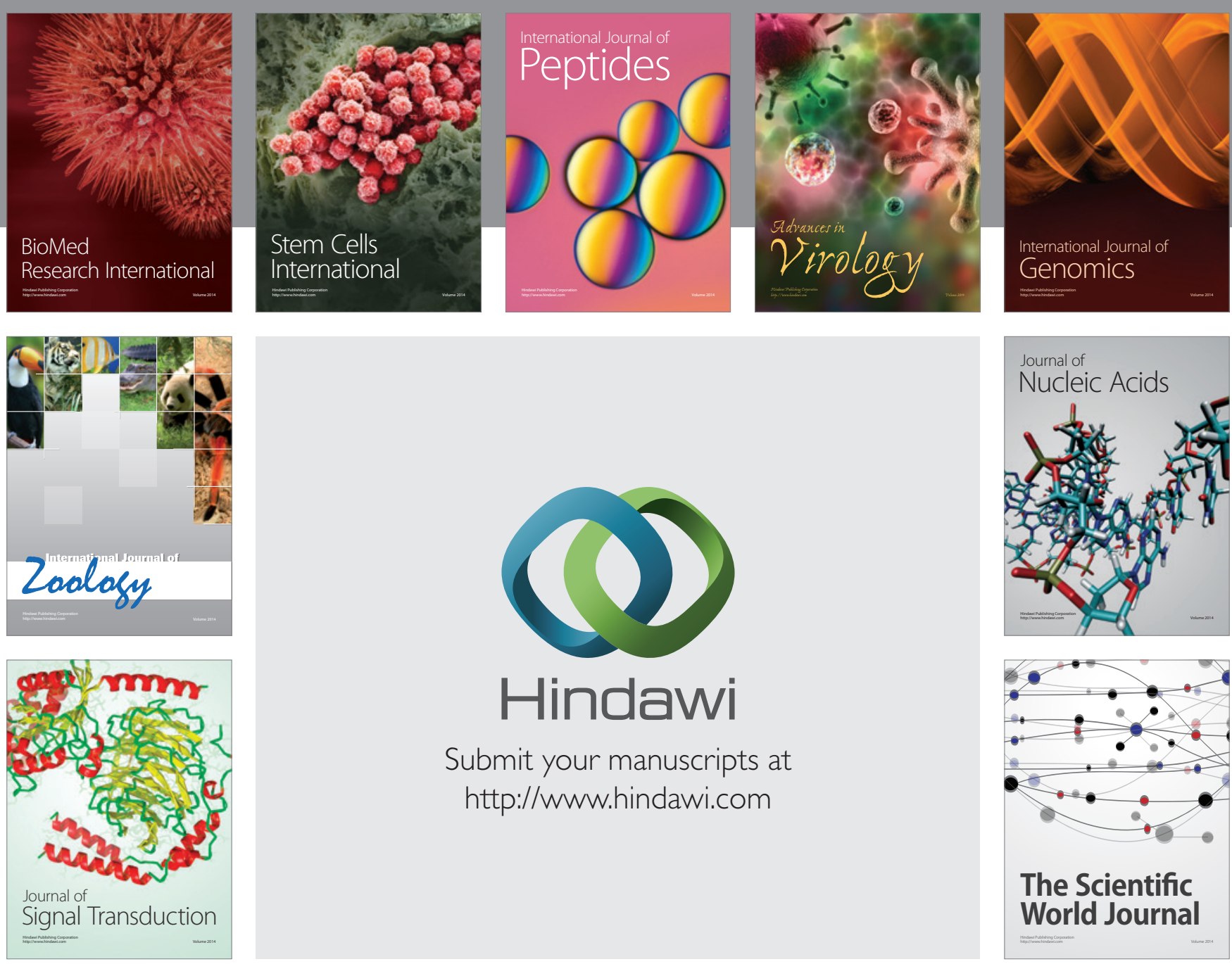

Submit your manuscripts at

http://www.hindawi.com
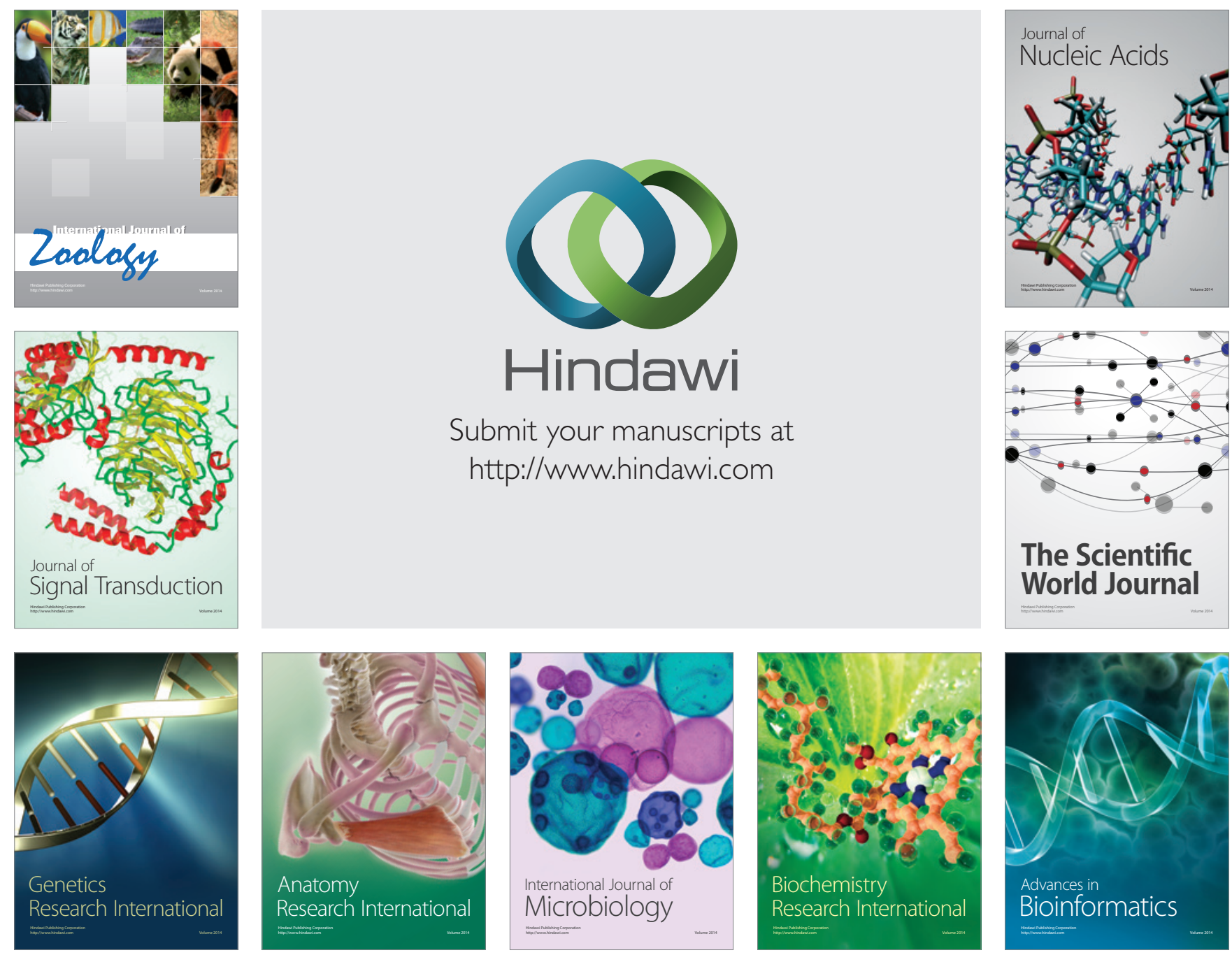

The Scientific World Journal
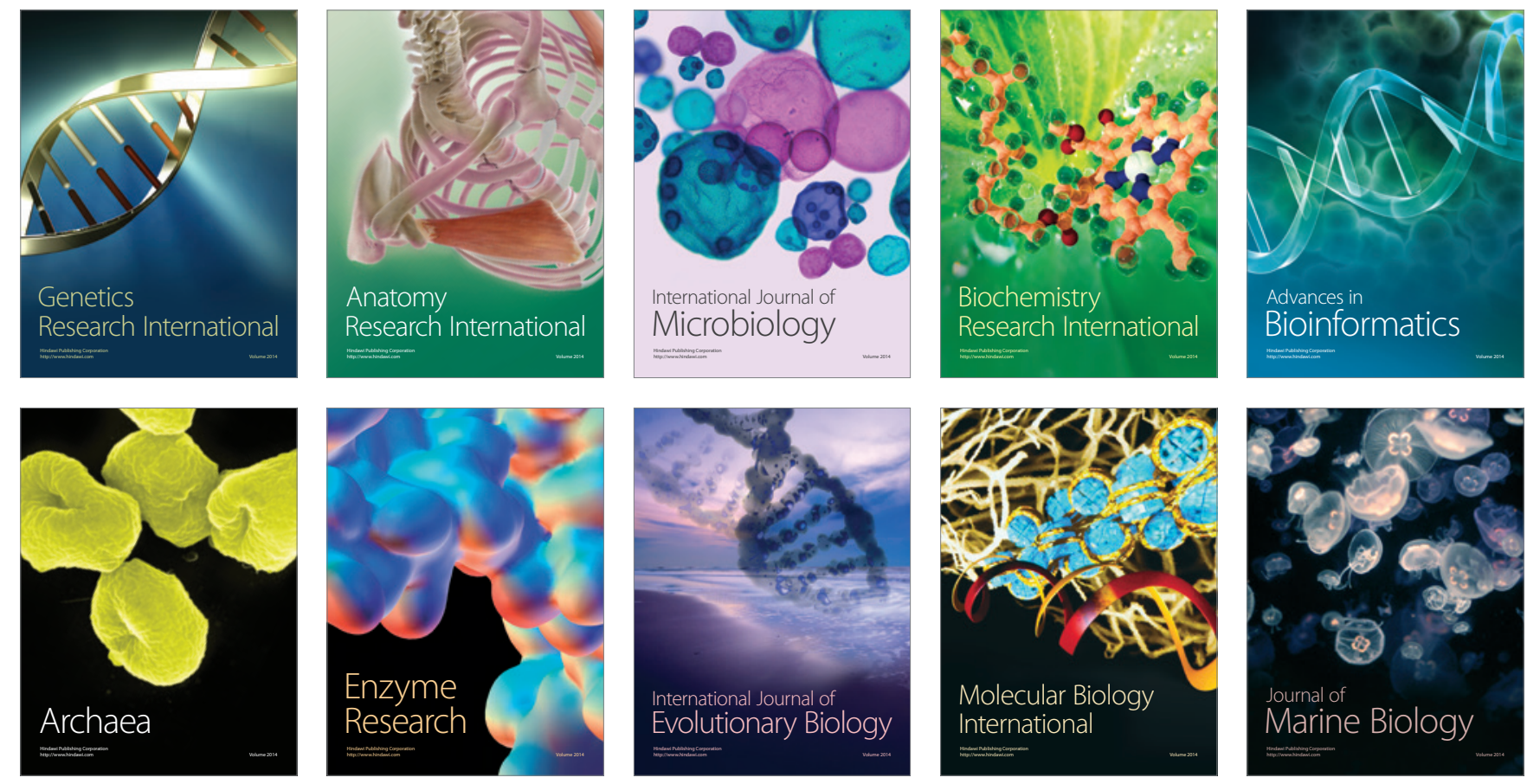